\title{
Association mapping of agronomically important traits in Russian collection of rapeseed
}

\author{
Gubaev R. ${ }^{1 *}$, Goryunova S. ${ }^{1,2}$, Goryunov D. ${ }^{1,3}$, Mazin P. ${ }^{1,4}$, Boldyrev S. ${ }^{1}$, \\ Chernova A. ${ }^{1}$, Ayupova A. ${ }^{1}$, Martynova E. ${ }^{1}$, Demurin Y. ${ }^{5}$, Mukhina Z. ${ }^{6}$, Khaitovich P. ${ }^{1}$ \\ ${ }^{1}$ Skolkovo Institute of Science and Technology, Moscow, Russia \\ ${ }^{2}$ Vavilov Institute of General Genetics, RAS, Moscow, Russia \\ ${ }^{3}$ Belozersky Institute of Physico-Chemical Biology, MSU, Moscow, Russia \\ ${ }^{4}$ Institute for Information Transmission Problems, RAS, Moscow, Russia \\ ${ }^{5}$ Pustovoit All-Russian Research Institute of Oil Crops, Krasnodar, Russia \\ ${ }^{6}$ All-Russian Rice Research Institute, Krasnodar, Russia \\ *e-mail: rim.gubaev@skoltech.ru,rimgubaev@gmail.com
}

Rapeseed (Brassica napus) is oilseed crop vastly used to produce vegetable oil for food, chemical, and biofuel industries. Despite the fact that rapeseed is quite popular in terms of trait-genotype association studies the Russian collections which are of high agricultural value remain poorly studied and described. Here we aim to map the agronomically important traits (lodging, plant height, glucosinolates content, time of flowering, seed mass, oil content) obtained for rapeseed collection from All-Russian Research Institute of Oil Crops (Krasnodar, Russia). In order to perform association mapping the information on abovementioned traits were obtained for 105 rapeseed lines. In order to perform genotyping, we applied restriction site DNA digestion procedure followed by Illumina high-throughput sequencing technique which allowed us to obtain around 8 million of paired-end reads for each plant sample in three replicates. Using GATK pipeline we have identified 761304 variants in total of which 221768 were presented by biallelic SNPs. After quality filtration procedure 8251 biallelic SNPs left which were used for downstream analysis. Population structure was revealed using admixture software which allowed to distinguish clear segregation of the rapeseed into two populations represented by winter and spring types of plant, respectively. The association mapping was performed in TASSEL software using mixed linear model approach. This allowed to reveal new potential genotype-phenotype associations. The results on loci significantly associated with glucosinolates content, plant height and flowering will be discussed in detail during the presentation.

Acknowledgements: This study was supported by the Ministry of Science and Higher Education of the Russian Federation (Grant No. 14.609.21.0099). 